\title{
A Scalable Mechanism Based on Blockchain for Information Processing in Energy Trading
}

\author{
Xunyan Jiang $\mathbb{D}^{1,2}$ and Lei $\mathrm{Wu} \mathbb{D}^{2}$ \\ ${ }^{1}$ School of Economics \& Management, \\ Hunan Provincial Engineering Research Center of Electric Transportation and Smart Distributed Network, \\ Changsha University of Science \& Technology, Changsha 410114, China \\ ${ }^{2}$ College of Mathematics and Computer, Xinyu University, Xinyu 338004, China \\ Correspondence should be addressed to Lei Wu; jxxywulei@126.com
}

Received 25 September 2020; Revised 25 October 2020; Accepted 29 October 2020; Published 16 November 2020

Academic Editor: Chin-Chia Wu

Copyright (C) 2020 Xunyan Jiang and Lei Wu. This is an open access article distributed under the Creative Commons Attribution License, which permits unrestricted use, distribution, and reproduction in any medium, provided the original work is properly cited.

\begin{abstract}
Energy Internet (EI) can provide a fair, transparent, and safe environment for the energy market through the rational use of the Internet and blockchain technology. However, the existing EI has complicated management in energy, capital, and information flow. Furthermore, blockchain technology (BT) is a new technique with a scalability problem. To overcome the problem, we proposed a scalable mechanism based on blockchain for information processing in energy trading. The proposed mechanism adopts three-way communication (TWC) between energy utilities and consumers to effectively manage the energy, capital, and information flow. In the running of TWC, three modules of BT-based Internet of Things (IoT) system, BT-based virtual currency system, and BT-based EI information platform are constructed. In addition, we proposed that these modules are managed by carrying out weak centralized management to make the EI system scalable. In the operation of this mechanism, the functions of the three modules are independent and parallel. The proposed mechanism can realize the efficient transmission of energy and information and help to solve the supervision problem of energy trading. Compared with the existing information process mechanism, it is superior in promoting the dynamic balance of resources in a wide range and meeting the fast, efficient, and safe energy trade needs between the supply and demand sides.
\end{abstract}

\section{Introduction}

According to the BP Statistical Review of World Energy 2015 [1], since 2010, China has been the world's largest energy consumer. For a long time, large centralized power plants use nonrenewable fossil fuels to generate power, which causes serious threats to energy security, environmental degeneration, climate change, and human health. To address the environmental energy dilemma [2], the best solution is to reduce energy consumption and integrate the increasing renewable energy sources (RES) into the current energy system. Nevertheless, energy efficiency needs to be taken into account. Energy efficiency can be achieved whenever volatile demands and renewable energy are managed. The existing energy-efficient power grid mainly is the smart grid
[3], where uncertainties and fluctuations in renewable energy management and volatile demands have always been concerned. Furthermore, existing wholesale markets lack a real-time response to the volatility and intermittent power generated by RES, and market prices do not reflect local energy shortages or oversupply [4]. In order to secure energy efficiency and support the reducing energy consumption and the integration of distributed RES into the energy system, new market approaches and information processing architectures should reflect the location of their services [5].

The locality of their services is manifested mainly by that consumers and prosumers trade self-produced energy on microgrid energy markets (MEM). The prosumers are referred to as the groups that consume and create energy. MEM allows consumers and prosumers to actively trade energy with small- 
scale participants in their community in real time [1]. MEM, on which energy trading is based, facilitates that energy is provided and consumed in a sustainable, reliable, and balanced way. If the microgrid is small for every household, the future energy network will be similar to the existing Internet. This network system is called Energy Internet (EI) [6]. The multilevel microgrid is the Energy Internet subsystem to achieve a twoway flow of energy and information. The EI extends the local energy trading to that of the big scale. Every family can create and use their own energy and exchange and share energy through the EI with other users. Therefore, EI provides a viable option to integrate the distributed RES economically into the current energy system [7, 8]. Moreover, investments in local generation of EI will be incentivized with the empowerment of small-scale-energy consumers and prosumers, which help to develop the microgrid communities with the self-sustainable characteristic and develop a stochastic dynamic programming model that cooptimizes the use of energy storage for multiple applications, such as energy, capacity, and backup services [9]. However, the implementation of a smart, secure, and innovative information system in EI is the key of the successful operation for EI [10, 11].

Blockchain technology (BT) [12], as a new database technology, has the advantages of decentralization, intelligent contract, cybersecurity, and privacy protection, etc. This technology provides transparent and user-friendly applications [13] in EI and is naturally suitable for the energy market [14]. At present, BT has come to the fore in the field of EI energy market. For example, in [15], the author proposed to manage the daily energy exchange through the application of a demand response framework based on decentralized cooperatives within a community of Smart Buildings. In the framework, a fully decentralized algorithm based on BT was used to trustily communicate, bill, and autonomously monitor via smart contracts among the participants. In $[16,17]$, the author proposed to manage transactions in the smart grid and supply chain members by using BT. The trusted communication and immutability of the transaction between generators and consumers were executed. In [18], the potential and applications of blockchain in the EI market as distributed renewable resources and smart grid networks were discussed, in which, the author proposed distributed and decentralized solutions to the future energy market. Blockchain technology has been adopted to solve the prevailing problems of existing EI, and demand response management, complex energy transactions, and data exchange, etc. can be automated by carrying out smart contracts. The above example shows that the projects based on BT and applied in EI have been developed. Among them, some research results were tested through the process of small-scale experiments in the industry at present.

Recently, in the academic world, several researchers have given attention to the conceptualization and implementation of blockchain-based EI energy markets. The study [1] proposed the concept of the BT-based MEM and built the framework for the BT-based MEM. This research validated that $\mathrm{BT}$ is a qualified technology for distributed MEM through simulating the performance of BT in Brooklyn microgrid. The study [19] studied the application of BT in the chemical industry by constructing a machine-to-machine electricity market. The article [20] used the decentralized traceability characteristics of BT to address the malicious actors in the carbon emissions trading system. That effectively confirmed that the application of BT in the carbon emissions trading system was feasible. The article [21] proposed the operation model and method of the decentralized distribution network to ensure the economic and cybersecurity of the power distribution network. It minimized the costs from eliminating the deviation of electricity through running multilateral trading of deviation power by bidding. The above research mainly focuses on the high cost and trust problems of centralized transaction management vulnerable to attack and the difficulty to guarantee user's privacy in the EI trade area. There are very few research articles on scalable information processing for the energy market of EI.

Based on the above analysis, BT is expected to achieve multiparty body automatic, reliable, fair, and real-time transactions in the EI system. However, BT has a scalability issue [22], and the EI system is complicated for the administration of energy, information, and capital flow. Furthermore, due to the insufficient consideration of the special attributes of energy goods, the EI system cannot promote the large-scale dynamic balance of resources and improve the efficiency of energy trading. To solve the problem, a scalable mechanism based on blockchain for information processing in energy trading is proposed. This mechanism constructs three modules: the BT-based Internet of Things (IoT) system, the BT-based virtual currency system, and the BT-based EI information platform to effectively manage energy, information, and capital flow. The three modules run in parallel, and their functions are independent. In this paper, we call this way as three-way communication (TWC) between merchants and consumers. In the process of TWC, to make the existing intelligent technologies be incorporated across the entire system, from energy generation, transmission, and distribution, to energy consumption at the customers' premises, the EI system was used to realize the open interconnection and coordination management of multienergy system. At the same time, with the aim of improving the reliability, efficiency, transparency, safety, and fair of the system, BT is the underlying technology. In addition, energy is different from general goods, when the BT-based energy trading lacks the supervision of the central organization, the determination of relevant standards can be solved through the implementation of weak centralization of energy transaction management. At the same time, the flows of energy, information, and capital are independent and need to be managed and run in parallel. As a result, a scalable information processing mechanism is implemented, which makes the energy exchange reasonable, secure, reliable, and flexible. Moreover, it can also promote a wide range of resource dynamic balance in the energy trading market, resulting in quick, efficient, and safe energy trading between supply and demand sides.

The contributions of our mechanism are summarized as follows: 
(1) A scalable information process mechanism is proposed to improve the performance of the EI system. This mechanism can supply a safe, fair, transparent, flexible environment for the energy markets.

(2) In this mechanism, the energy, capital, and information flow in EI are addressed, respectively, by the TWC approach. In the TWC approach, we constructed three modules: the BT-based IoT system, the BT-based virtual currency system, and the BT-based EI information platform to improve the efficiency of energy trading.

(3) In energy trading, we consider the characteristic of energy. Each energy trading was audited through weak centralization management. The combination of the weak centralization management and the TWC approach makes the EI system scalable.

\section{Relevant Work}

In this section, the relevant theories for the proposed mechanism are described, including the EI energy market, blockchain technology, and weak centralized management.

2.1. EI Energy Market. The traditional energy market is mainly composed of exchange transactions and over-the-counter (OTC) [23]. In exchange transaction mode, market participants usually include users, central institutions, and third parties. Energy trading is planned and managed by the exchange center which is responsible for the overall balance of the energy market. Third-party institutions perform ratings, insurance, trust and financing, transaction clearing, and other types of work. Therefore, energy trading in the exchange mode will generate high third-party costs. Trading data is easy to be lost or tampered with, and user privacy is difficult to guarantee. Furthermore, trading information is asymmetric. Frequent information proofreading and high time cost are not conducive to efficient real-time transactions in EI. In OTC transaction mode, there is no fixed place to trade and there are no restrictions on market participants. Market makers are responsible for marketing organizations. Buyers and sellers negotiate prices outside the exchange through telephone and computer networks. These transactions are carried out by agents of market makers, and there is no limit to the number and units of transactions. Trading is basically a direct transaction between buyer and seller, and the price does not include the trading commission. However, the market maker who sets the bid-ask price will get a certain amount of money from the bid-ask spread he sets. Therefore, OTC transactions add additional market costs to energy trading.

EI has introduced the concept of the Internet on the basis of the traditional energy network. It has new connotations which are user-centered and distributed peer-to-peer sharing [24]. In EI, different energy industries are interconnected and managed in a coordinated way, many of which are connected to distributed renewable energy sources such as wind, solar, and tidal power. In the future, EI will cover a large number of users who may be consumers or prosumers. At the same time, energy trading will be characterized by diversification of subjects, diversification of commodities, decentralization of decision-making, transparency of information, and timeliness [25]. The new energy structure will be based on the distributed power generation technology, microgrid technology, smart grid technology [26-28], etc., and the energy production, manufacture, storage, and transportation will be coordinated and managed in EI. It is expected to reach comprehensive energy trading, including power transactions, primary energy transactions, and auxiliary power services transactions [23]. As a result, in the energy market under the EI environment, the user can voluntarily choose the type of energy service on the basis of the self-load characteristic, with the power exchange as the center. The transaction participants can independently decide whether to issue or purchase energy according to different energy service types in EI.

2.2. Blockchain Technology. BT, which is introduced as the underlying technology of bitcoin, is a database technology without a dedicated central authority to manage data $[12,29]$. In a broader field of economic applications, the use of BT is called a verification mechanism for cryptocurrency. The blockchain of BT is a kind of data structure linked by chronological order $[30,31]$. In this structure, blocks are the basic units of forming a blockchain, and relevant information and records are stored in blocks. Each block consists of a header and a body, where the header is used to link with the previous block. The body is used to record information. The network of blockchain is a peer-to-peer (P2P) network $[32,33]$, which has no central server and router, encapsulating specific communication protocols and data verification mechanisms. In the network, each node has a peer status and saves all the data information of the whole blockchain. All participants distributed in different locations jointly own the right of management and supervision data. Therefore, BT has the characteristics of decentration, transparency, and traceability.

The core technologies of blockchain include asymmetric encryption algorithm [34] and smart contracts $[35,36]$. The former uses public key and private key to solve the problem of user information security and trust. Each participant has his own public and private key. After using one of the keys that encrypted information, only one other corresponding key is used to unlock it. The smart contract is a kind of digital contract similar to business rules, which stipulates the obligations of each party to fulfill and the judgment conditions of contract execution. For users, smart contracts are usually considered an automatic security account, whose code and status are in BT. Therefore, in an information system based on BT, it is expected to reach complete decentralization by using cryptographic security, smart contracts, and a decentralized consensus mechanism. For all this, BT still has some problems, such as scalability issues, limited transaction loads, and its complex technical protocol and implementation [22]. Nevertheless, BT is an emerging technology with the potential to provide new ways to benefit various systems. 
2.3. Weak Centralized Management. Chai et al. [23] suggested that energy with different physical properties, which is the national strategic resources, is related to the national economy and people's livelihood. Therefore, energy trading is different from ordinary commodity trading. It is essential to give certain regulatory measures about the access and exit of participants of the energy market, the process of transaction, etc. In addition, it is difficult to solve the problem completely when there are disputes in the transaction only by the system itself and the enforcement of intelligent contracts. The system, whose trading is completely decentralized, is not suitable for the energy market. Some supervision for the management of energy transactions based on BT is needed, which is called weak centralized management.

Weak central management is implemented by weak central institutions, which have the right to manage accounts and supervise transactions. It is a special node in the blockchain trading network. This node is different from the nodes which participate in the process of energy trading game and independently completes the determination of energy price and transaction records. The node is responsible for such work as confirmation of transaction qualification and contract review. It does not have the right to store energy transaction information and modify the information. Relevant modifications are subject to the consent of the nodes involved in energy trading. Therefore, in the implementation of a weak central management system for energy trading, weak central agencies should audit user qualifications and approve the right users obtained to register. After registering, the users' information is broadcast to all network nodes of the P2P network. When all the nodes reach a consensus, account registration is successful, and access to energy transactions is allowed. All the transaction information generated by these users shall be checked by the weak central institution so that the legitimate transaction can be broadcast to all network nodes. At the same time, transaction information can be stored in the blockchain. If users exit the energy market, the notification of exit will be issued in the whole network. After the nodes in the network reach consensus, the central institution will cancel the registration information in time.

\section{The Proposed Mechanism}

In this section, based on BT, we derive TWC for the efficient operation of the EI market. The aim of the TWC is to overcome the shortcomings of $\mathrm{BT}$ to make the information system scalable with a high trading volume of business. A schematic diagram of the EI based on TWC is given in Figure 1. In the framework, the source, grid, load, and reserve are composed of the modules of the BT-based EI energy market. The modules of the market are closely integrated and coordinated with the administration of the EI system. In virtue of energy technologies such as energy production, transmission, distribution, and use, the production, conversion, reserve, and other links can effectively execute scheduling, controlling, managing, and using in the source, grid, load, and reserve. In these modules, the source represents the traditional energy supply, distributed energy which mainly includes RES. These energies are transported and distributed to industrial parks, business enterprises, and individual users by energy transmission and distribution systems. Reserves refer to battery energy, capacitive energy, and pumped storage which are produced by energy enterprises, communities, or prosumers. These energies can be supplied to users through energy storage control technology. Moreover, the energies in sources and reserves can be transformed for heat, electricity, and cold energy. The devices, which derive from source, grid, load, and reserve, are interlinked by IoT technology to form the physical network of EI. Considering that there is a large amount of personal data in IoT, and there is arguably no single point of failure or vulnerability in blockchain, to strengthen cybersecurity and protect privacy, this paper proposes that the storage of energy information derived from the devices is based on BT to form the energy flow (shown in Figure 1 with green thread). BT-based IOT is responsible for the management of the energy flow like the logistics companies, whose functions are independent. In Figure 1, the red line represents the capital flows which derive from the financial transactions between buyers and sellers, e.g., clearing information, the pattern of payment, payment amount, etc. Taking into account the BT-based bitcoin system, which is a virtual currency system, it has the advantage of distributed accounting. To construct a multisignature-protected decentralized marketplace, this paper proposes the BT-based administration of capital flow. In this way, user information will not be managed and stored. Even if a hacker successfully breaches the BT-based EI system, the private key of the virtual wallet cannot be accessed. Another blue line represents the information flow generated by communication between energy utilities and consumers, such as proof of identity for individuals as well as assets, user information, and system operation status, since the running of energy and capital flow can produce information from energy and finance trading. To effectively manage the information, we propose that the role of administration for information flow is played by the BT-based EI information platform. The platform can implement integrated information from the energy market for the user to access. Based on the above description, the energy, capital, and information flow are three breaches of the BT-based EI system, respectively. In this paper, we call the approach for the three breaches TWC. The TWC-based scalable information process mechanism will be described in detail.

3.1. TWC-Based EI. The specific design for the building elements of various blockchains can vary according to the specific use case. For the BT-based EI energy market, it has a distributed software architecture that allows for a complete and continuously tracking of even the smallest energy transactions. Its main advantages are transparent, distributed, and secure transaction logs. However, it has scalability issues. Furthermore, a certain amount of information confirmation cost has to be generated in the process of energy trading creation. Thus, as an information system, a 


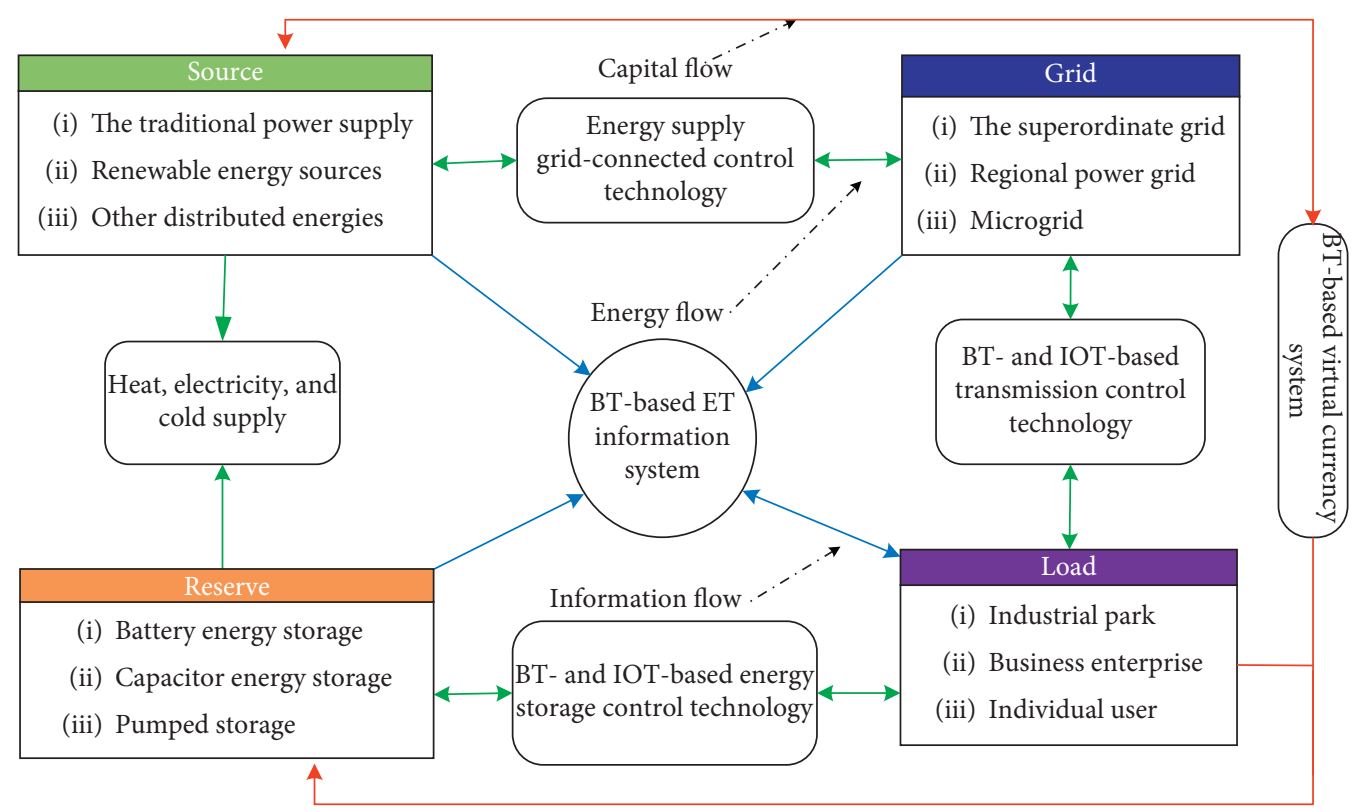

FIgURE 1: A schematic diagram of TWC-based EI.

trade-off between high information accuracy and storage mode needs to be considered. To improve the scalability of the BT-based EI system and the efficiency of energy trading, we believe that the market participants in EI will be a less costly hash-based user authentication mechanism. However, the level of security and resilience required for efficient integration of distributed energy resources may not be ensured through proof-of-identity authentication mechanisms. Therefore, we propose a TWC-based EI energy trading system. In the system, there are three ways to manage this information flow. One way is to correct and store information by the BT-based IoT system. The second way is to address financial transactions by BT-based virtual currency system. The third way is to manage trading information by BT-based EI information platform.

3.2. BT-Based IoT System. In the traditional EI system, the traditional IoT has a data center, which collects information from the connected devices. Furthermore, the information derived from devices is stored in a centralized cloud model. This way may become even more problematic and potentially risky as the number of network nodes increases. For example, some smart devices violate personal privacy, and government security agencies can review data stored in central servers without authorization [37]. Operators can also sell users' private data to advertising companies for commercial profit [38]. Blockchain provides a good way to solve these problems through promoting collaboration between transaction processing and interactive devices in the decentralized IoT. Moreover, the IoT can be scaled up to ensure privacy, security, and the establishment of untrusted transactions. Thus, the BT-based IoT approach would be more suitable and more effective for managing energy information in the EI energy market.
In IoT, the interconnection devices are several feet apart from each other; the connection between them needs to go through EI. Most devices of IoT send data to EI and messages can be sent from the EI to the devices, where processing and storage are often carried out. If a device connected to a server is hacked, it can disrupt the whole network. On the other hand, it is difficult for the data center to ensure the validity and management of data. The BT-based IoT system can ensure that the good and proper use of information and the message exchanges between devices can be treated as financial transactions in a bitcoin network. From Figure 1, the energy information in the model of source, grid, load, and reserve is sent through the blockchain to verify these cryptographic signatures to ensure that only the originator of the message could send it. At the same time, the messages are exchanged through the blockchain cryptographically sign transactions. Thus, when energy trading with security holes is not returned, the owner of the device can be tracked and easily contacted.

In the EI system, the devices of IOT mainly include energy router, energy exchange, and energy interface. These devices connect the server as the back end in the EI system. Among them, energy routers implement the communication connection with the back end. Energy exchanges supply energy information for the subnet, such as total input, output, and storage of electrical energy; total generation, consumption, and storage of thermal energy; and storage and consumption of other forms of energy. Energy interfaces are the port of transportation, petrochemicals, and other networks. Among them, various distributed renewable energy, devices for generation of power and heating, and distributed energy storage devices join up EI to achieve the identification of the distributed devices, the detection of the status of the distributed devices, and various forms of energy calibration. The schematic diagram of the BT-based IoT 
system is shown in Figure 2. From Figure 2, the accessing devices join up the EI through the devices of IoT to construct the subnet of IoT. All subnets consist of the physical networks of EI. Its operation achieves distribution and management of energy flow, safe and reliable network operation, efficiency, and economy of energy use.

3.3. BT-Based EI Information Platform. In Section 3.2, the BT-based IoT system, which constitutes the physical network of EI, is described. The energy information flows EI energy market that combines BT-based virtual currency system and BT-based EI information platform to produce energy trading information, financial information, and communication information, all of which are accessed and used by the user in BT-based EI information platform. This process is implemented by the virtual network of EI. The schematic diagram of the virtual network of EI is shown in Figure 3. From Figure 3, the energy flow is represented by green threads. The capital flow (shown in Figure 3 with red threads) was managed by a BT-based virtual currency system. The communication information flow is shown with blue threads.

In the EI energy market, users incarnate the sellers and buyers, namely, the participants, such as energy traders, energy enterprises, and energy agents. In BT, smart contracts have the characteristic of the absence of accountability. The subject of energy trading is often a virtual account, and there are a series of ethical problems to be solved. If users want to $\log$ in to the EI energy market by registering at the EI information platform, the identity of the user needs authentication. Therefore, we proposed to introduce a "digital identity authentication service" which is based on BT. At the same time, the process of authentication is implemented as weak center data management and provides different kinds of digital identity authentication services. That is to say, the third-party organization, which proves the personal details, but does not store the user information, undertakes the regulatory task to audit the information of participants. In this way, it is to ensure the rationality and legitimacy of the energy traders.

Based on the above analysis, the authorized users enter the EI energy market for energy trading. In this platform, users can not only order energy from the energy traders or sell energy to the energy traders but also buy/sell energy to each other. When they conduct energy trading, they trust each other by a consensus mechanism derived from a certain mathematical algorithm. Moreover, the authentication of energy information follows the data encryption principle of blockchain technology. The distributed "bookkeeping" based on blockchain and metrological certification which is authenticated by all nodes of the whole system can guarantee the authority of measurement and certification for energy information. Therefore, the energy information which is produced by the BT-based IoT system is safe and reliable. Furthermore, since the smart contracts can guarantee the unification and synchronization of the control modes, control interval time, and signal instructions of multienergy systems with different physical characteristics and scheduling modes, energy trading can be automatically accomplished the underlying communication between buyers and sellers by means of BT-based EI information platform. However, considering that the energy commodities are different from general goods, energy trading cannot be completely out of the country's credit endorsement. It needs safeguards and supervision of policy. Thus, when energy derived from the electric power system, heating system, gas system, etc. enters the energy market to participate in trading, third-party organizations are needed to audit the trading information. In energy trading, the thirdparty organization is the node of EI, but it does not have the jurisdiction to store the trading information. In financial trading, the information on energy trading is used for clearing transactions. Users can freely choose the means of payment according to the actual situation and preferences and complete the receiving and payment transactions in the BT-based EI information platform.

3.4. BT-Based Virtual Currency System. From Figure 3, the virtual currency is issued, circulated, and traded in the system. The financial trading between buyers and sellers is based on a BT-based virtual currency system. At the same time, this system achieves the issuance and renewal of virtual currency, and the circulation of virtual currency can be driven by energy trading in the BT-based EI energy market. Energy participants play the role of each network node of virtual currency. They actively engage in energy trading to compete with the award by a game approach which ensures a stable virtual currency consensus and security. The system of virtual currency issues a certain number of virtual currencies for a new block to reward the creator. To establish revenue sharing fees, partial participants can cooperate with each other to play the game together. Thus, after the issuance of the virtual currency, it goes into circulation. Users who own virtual currency can pay virtual currency for energy and services through the BT-based EI information platform. It is worth noting that this virtual currency is different from the bitcoin, its value is steadfast, and its attributes are various at different times. As we all know, the blockchain has the characteristic of timestamps. This paper proposes that the issuance and renewal of virtual currency are based on blockchain and the virtual currency can have the characteristic of timestamp. We give the different attributes of virtual currency in view of its different issuance times. For example, to obtain different amounts of virtual currency as incentives, buyers purchase various energies at different times. At the same time, the virtual currency with various attributes has different operating life.

In view of the above analysis, how much energy participants gain to depend on the outcome of the game for energy trading. The game winner gains and gets the right to pack the blocks. In addition, because of the flexibility of the energy market, investors or speculators can carry out virtual currency trading based on BT-based virtual currency systems. Such trading can be developed between the participants of energy trading. The financial institutions can take part in the virtual currency as participants to promote the 


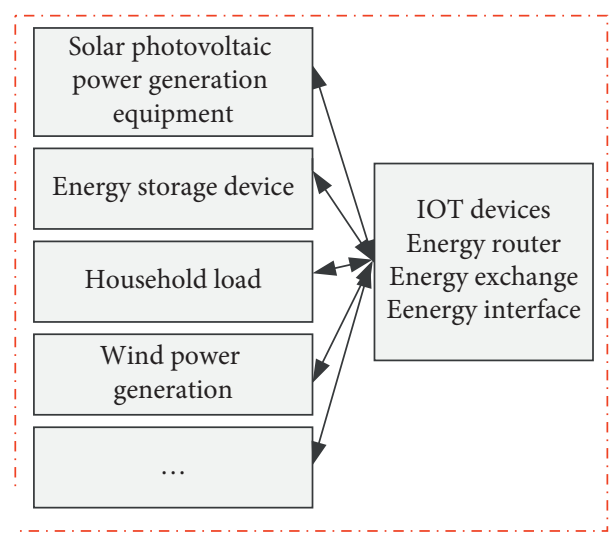

(a)

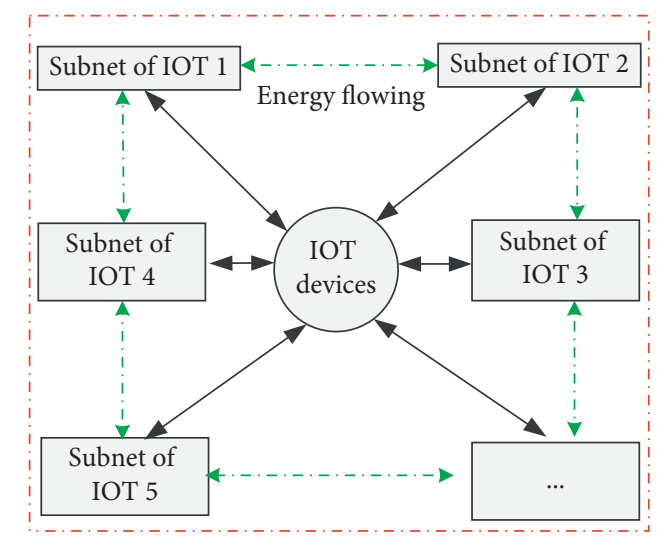

(b)

Figure 2: The schematic diagram of the BT-based IoT system. (a) Subnet of IoT. (b) The physical network of EI.

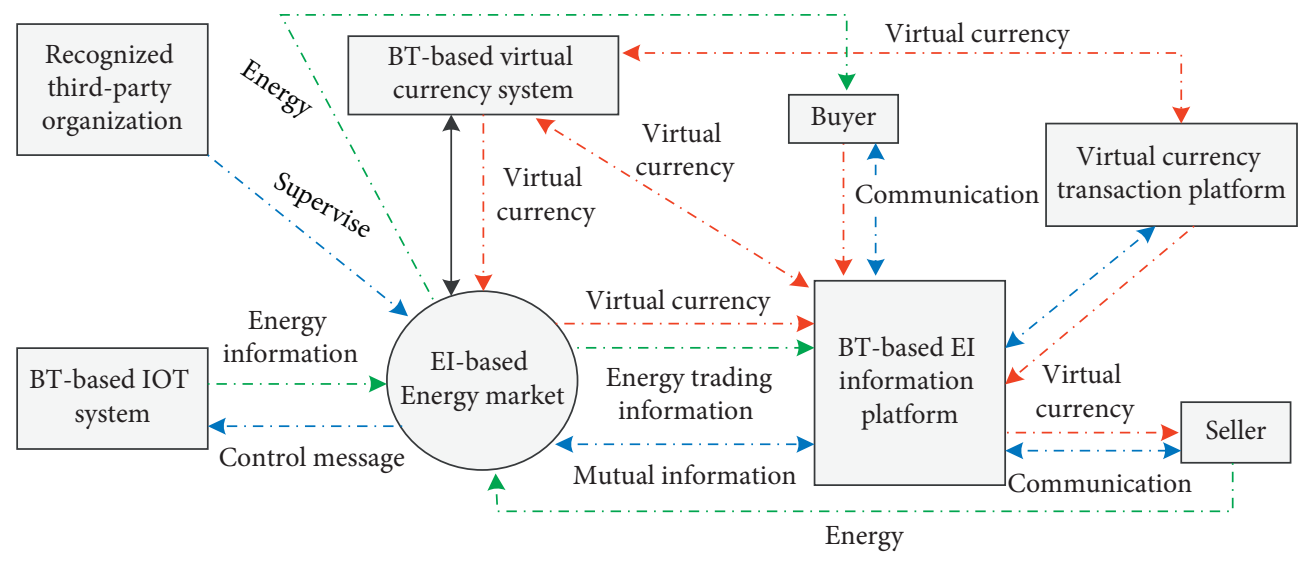

FIGURE 3: The schematic diagram of the BT-based virtual currency system.

development of the energy market. Regardless of financial trading, after the transaction is completed, each transaction will be validated by all nodes in the BT-based EI and recorded in blocks. follows:

The main contributions of this paper are summarized as

(1) We present an overview of the literature for the EI energy market, BT, weak central management, and their combination.

(2) Based on this existing literature, we proposed to construct three modules of BT-based IoT system, BT-based virtual currency system, and BT-based EI information platform for the efficient design and operation of BT-based EI energy market.

(3) Thus, we developed the TWC approach and modulebased weak central management to improve the scalability of the EI system.

However,

(4) The TWC approach which is based on three modules still needs to be tested and adequately adapted for the future energy market. The implementation of the
TWC approach requires further development to adequately implement intelligent supply-demand response strategies.

(5) The regulation of weak centers needs to be adapted for allocation efficiency. The corresponding smart contracts seem to need different departments to formulate.

\section{Conclusion}

This paper presents a scalable mechanism based on blockchain for information processing in energy trading. In the mechanism, a communication approach (called TWC) between merchants and consumers is proposed to deal with energy trade by incorporating the existing intelligent technologies, especially, the BT technology, across the entire system. We have derived three modules (including BT-based IoT system, BT-based virtual currency system, and BT-based EI information platform) and a weak centralized management mechanism to implement TWC. In the process of TWC, the problem of energy flow was addressed by a BTbased IoT system. This system can avoid the whole network to be disrupted because the simple notes are hacked. At the 
same time, because of the characteristics of BT, it strengthens cybersecurity and privacy protection and ensures the correctness of data. In addition, the capital flow is managed through a BT-based virtual currency system. This system is like the bitcoin system, but it is different. In this study, the system launches and updates virtual currencies with different attributes at different times, to improve the efficiency of the energy market. Furthermore, the communication information is administrated by a BT-based EI information platform. In this platform, buyers and sellers have completed the auditing of identity authentication from the third-party organizations. The authorized users can use the game strategy to carry out energy trading. As a result, the information on energy trading is used for clearing to generate financial information. Finally, the winner in this game gets the right to package the information into a blockchain and broadcast it to all nodes in the network. With all that, we put forward that there are several advantages of the proposed mechanism. At the same time, the current type of BT will promote various industrial and academic projects in the EI energy market. The project's findings need to be further tested to evaluate economic and socioeconomic impacts. Thus, it is hoped that this study can provide a reference for researchers of BT-based EI.

\section{Data Availability}

The corresponding data used to support the findings of this study are included within the article.

\section{Conflicts of Interest}

The authors declare that they have no conflicts of interest.

\section{Acknowledgments}

This research was supported by the National Natural Science Foundation of China (No. 71420107027).

\section{References}

[1] E. Mengelkamp, J. Gärttner, K. Rock, S. Kessler, L. Orsini, and C. Weinhardt, "Designing microgrid energy markets: a case study: the Brooklyn Microgrid," Applied Energy, vol. 210, pp. 870-880, 2018.

[2] L. Tianguang and A. Qian, "Interactive energy management of networked microgrids-based active distribution system considering large-scale integration of renewable energy resources," Applied Energy, vol. 163, pp. 408-422, 2016.

[3] J. Vardakas, N. Zorba, and C. Verikoukis, "A survey on demand response programs in smart grids: pricing methods and optimization algorithms," IEEE Communications Surveys \& Tutorials, vol. 17, pp. 607-178, 2015.

[4] A. Monacchi and W. Elmenreich, "Assisted energy management in smart microgrids," Journal of Ambient Intelligence and Humanized Computing, vol. 7, no. 6, pp. 901-913, 2016.

[5] A. Nieße, S. Lehnhoff, M. Tröschel et al., "Market-based selforganized provision of active power and ancillary services: an agent-based approach for smart distribution grids," Aachen, Germany: IEEE Complexity in Engineering (COMPENG), pp. 1-5, 2012.
[6] A. Ahl, M. Yarime, K. Tanaka, and D. Sagawa, "Review of blockchain-based distributed energy: implications for institutional development," Renewable and Sustainable Energy Reviews, vol. 107, pp. 200-211, 2020.

[7] V. Lesic, A. Martinčević, and M. Vasak, "Modular energy cost optimization for buildings with integrated microgrid," Applied Energy, vol. 197, pp. 14-28, 2017.

[8] P. Kou, D. Liang, and L. Gao, "Distributed EMPC of multiple microgrids for coordinated stochastic energy management," Applied Energy, vol. 185, pp. 939-952, 2017.

[9] V. N. Coelho, M. Weiss Cohen, I. M. Coelho, N. Liu, and F. G. Guimarães, "Multi-agent systems applied for energy systems integration: state-of-the-art applications and trends in microgrids," Applied Energy, vol. 187, pp. 820-832, 2017.

[10] R. Schleicher-Tappeser, "How renewables will change electricity markets in the next five years," Energy Policy, vol. 48, pp. 64-75, 2012.

[11] K. Boroojeni, M. H. Amini, A. Nejadpak, T. Dragicevic, S. S. Iyengar, and F. Blaabjerg, "A novel cloud-based platform for implementation of oblivious power routing for clusters of microgrids," IEEE Access, vol. 5, pp. 607-619, 2017.

[12] T. McGhin, K.-K. R. Choo, C. Z. Liu, and D. He, "Blockchain in healthcare applications: research challenges and opportunities," Journal of Network and Computer Applications, vol. 135, pp. 62-75, 2019.

[13] M. Yang, T. Zhu, K. Liang, W. Zhou, and R. H. Deng, "A blockchain-based location privacy-preserving crowdsensing system," Future Generation Computer Systems, vol. 94, pp. 408-418, 2019.

[14] N. Zhang, Y. Wang, C. Q. Kang, J. N. Cheng, and D. W. He, "Blockchain technique in the energy internet: preliminary research framework and typical applications," Proceedings of the CSEE, vol. 36, pp. 4011-4022, 2016.

[15] O. V. Cutsem, D. H. Dac, P. Boudou, and M. Kayal, "Cooperative energy management of a community of smartbuildings: a Blockchain approach," Electrical Power and Energy Systems, vol. 117, pp. 1-11, 2020.

[16] M. Li, S. J. Shao, Q. W. Ye, G. Y. Xu, and G. Q. Huang, "Blockchain-enabled logistics finance execution platform for capital-constrained E-commerce retail," Robotics and Computer Integrated Manufacturing, vol. 65, pp. 1-14, 2020.

[17] Y. F. Li, B. Wang, and D. Yang, "Research on supply chain coordination based on block chain technology and customer random demand," Discrete Dynamics in Nature and Society, vol. 2019, Article ID 4769870, 2019.

[18] A. Miglani, N. Kumar, V. Chamola, and S. Zeadally, "Blockchain for internet of energy management: review, solutions, and challenges," Computer Communications, vol. 151, pp. 395-418, 2020.

[19] J. J. Sikorski, J. Haughton, and M. Kraft, "Blockchain technology in the chemical industry: machine-to-machine electricity market," Applied Energy, vol. 195, pp. 234-246, 2017.

[20] K. N. Khaqqi, J. J. Sikorski, K. Hadinoto, and M. Kraft, "Incorporating seller/buyer reputation-based system in blockchain-enabled emission trading application," Applied Energy, vol. 209, pp. 8-19, 2018.

[21] Z. Li, S. Bahramirad, A. Paaso, M. Yan, and M. Shahidehpour, "Blockchain for decentralized transactive energy management system in networked microgrids," The Electricity Journal, vol. 32, no. 4, pp. 58-72, 2019.

[22] R. Beck, J. S. C. Stenum, N. Lollike, and S. Malone, "Blockchain-the gateway to trust-free cryptographic transactions," in Proceedings of the European Conference on Information Systems, pp. 1-14, Istanbul, Turkey, June 2016. 
[23] J. Q. Cai, S. X. Li, B. Fan, and L. R. Tang, "Blockchain based energy trading in energy internet," Electric Power Construction, vol. 38, pp. 24-31, 2017.

[24] C. Park and T. Yong, "Comparative review and discussion on P2P electricity trading," Energy Procedia, vol. 128, pp. 3-9, 2017.

[25] J. Reynolds, Y. Rezgui, and J.-L. Hippolyte, "Upscaling energy control from building to districts: current limitations and future perspectives," Sustainable Cities and Society, vol. 35, pp. 816-829, 2017.

[26] W. P. Zarakas, "Growth prospects and shifting electric utility business models: retail, wholesale and telecom markets," The Electricity Journal, vol. 28, no. 5, pp. 59-67, 2015.

[27] C. Zhang, J. Wu, M. Cheng, Y. Zhou, and C. Long, "A bidding system for peer-to-peer energy trading in a grid-connected microgrid," Energy Procedia, vol. 103, pp. 147-152, 2016.

[28] E. Melville, I. Christie, K. Burningham, C. Way, and P. Hampshire, "The electric commons: a qualitative study of community accountability," Energy Policy, vol. 106, pp. 12-21, 2017.

[29] L. Zhong, Q. Wu, J. Xie, J. Li, and B. Qin, "A secure versatile light payment system based on blockchain," Future Generation Computer Systems, vol. 93, pp. 327-337, 2019.

[30] P. Rogaway and T. Shrimpton, "Cryptographic hash-function basics: definitions, implications, and separations for preimage resistance, second-preimage resistance, and collision resistance," Fast Software Encryption, vol. 3017, pp. 371-388, Springer Berlin Heidelberg, New York, USA, 2004.

[31] Q. Feng, D. He, S. Zeadally, M. K. Khan, and N. Kumar, “A survey on privacy protection in blockchain system," Journal of Network and Computer Applications, vol. 126, pp. 45-58, 2019.

[32] A. Savelyev, "Copyright in the blockchain era: promises and challenges," Computer Law \& Security Review, vol. 34, no. 3, pp. 550-561, 2018.

[33] M. Muzammal, Q. Qu, and B. Nasrulin, "Renovating blockchain with distributed databases: an open source system," Future Generation Computer Systems, vol. 90, pp. 105-117, 2019.

[34] L. Diestelmeier, "Changing power: shifting the role of electricity consumers with blockchain technology - policy implications for EU electricity law," Energy Policy, vol. 128, pp. 189-196, 2019.

[35] S. E. Chang, Y.-C. Chen, and M.-F. Lu, "Supply chain reengineering using blockchain technology: a case of smart contract based tracking process," Technological Forecasting and Social Change, vol. 144, pp. 1-11, 2019.

[36] X. Wang, W. Yang, S. Noor, C. Chen, M. Guo, and K. H. van Dam, "Blockchain-based smart contract for energy demand management," Energy Procedia, vol. 158, pp. 27192724, 2019.

[37] Z. Guan, X. Lu, W. Yang, L. Wu, N. Wang, and Z. Zhang, "Achieving efficient and Privacy-preserving energy trading based on blockchain and ABE in smart grid," Journal of Parallel and Distributed Computing, vol. 147, pp. 34-45, 2021.

[38] S. Zhang, J. Rong, S. Noor, and B. Wang, "A privacy protection scheme of smart meter for decentralized smart home environment based on consortium blockchain," Electrical Power and Energy Systems, vol. 121, pp. 1-10, 2020. 